\title{
Visualization of Leukocyte Transendothelial and Interstitial Migration Using Reflected Light Oblique Transillumination in Intravital Video Microscopy
}

\author{
Thorsten R. Mempel Christian Moser Joerg Hutter Wolfgang M. Kuebler \\ Fritz Krombach
}

Institute for Surgical Research, University of Munich, Munich, Germany

\section{Key Words}

Intravital microscopy · Cremaster muscle, mouse • Inflammation - Leukocyte transendothelial migration • Cell migration - Oblique illumination

\begin{abstract}
Dynamic visualization of the intravascular events leading to the extravasation of leukocytes into tissues by intravital microscopy has significantly expanded our understanding of the underlying molecular processes. In contrast, the detailed observation of leukocyte transendothelial and interstitial migration in vivo has been hampered by the poor image contrast of cells within turbid media that is obtainable by conventional brightfield microscopy. Here we present a microscopic method, termed reflected light oblique transillumination microscopy, that makes use of the optical interference phenomena generated by oblique transillumination to visualize subtle gradients of refractive indices within tissues for enhanced image contrast. Using the mouse cremaster muscle, we demonstrate that this technique makes possible the reliable quantification of extravasated leukocytes as well as the characterization of morphological phenomena of leukocyte transendothelial and interstitial migration.
\end{abstract}

Copyright $\odot 2003$ S. Karger AG, Basel

\section{Introduction}

The extravasation of leukocytes into tissues in the context of inflammation, immune response, or homeostatic trafficking is a multistep process that is governed by a series of signaling, adhesive, and migratory events which in concert facilitate the targeted, regulated recruitment of these cells to specific sites in the body [1]. In the past, brightfield and fluorescence intravital microscopic observation of the initial events in this process such as leukocyte rolling along and firm adhesion to the vessel wall has provided information which allowed for an integrated view on the underlying molecular mechanisms [2]. Although descriptive or quantitative assessment of leukocyte extravasation or of interstitial migration has been reported in tissues such as the rabbit ear [3], hamster cheek pouch [4], rat [5] and mouse [6] mesentery or cremaster muscle [7], the discrimination of morphological details has so far been limited by the poor image contrast of cells obtainable by brightfield microscopy within a turbid medium such as the interstitium. Since the visualization of phase gradients within unstained specimens, as realized by phase contrast [8], differential interference contrast [9] or Hoffman modulation contrast [10] microscopy, enables the study of dynamic cellular events in vitro, we hypothesized that the application of the underly-
Dr. Fritz Krombach

Institute for Surgical Research, University of Munich

Marchioninistrasse 27, DE-81377 Munich (Germany)

Tel. +49 897095 4359, Fax +49 8970954353

E-Mail krombach@icf.med.uni-muenchen.de 
ing optical principles to intravital microscopy might also enable the study of cellular events during and following leukocyte transendothelial migration. We therefore designed a microscopy setup which employs the principle of oblique transillumination [11] by placing a tilted reflector directly underneath a translucent specimen and transilluminating the tissue by a beam of near-monochromatic, near-infrared light. After its reflection, the beam transmits the specimen in a direction oblique to the axis of the detection optics. The resulting exclusion of one sideband of diffracted light allows for constructive and destructive interference to occur at the image plane, converting previously invisible gradients of refractive index within the specimen into intensity gradients in the image. To demonstrate the potential usefulness of this method, we provide quantitative data describing the effects of deficiency in either P-selectin or intercellular adhesion molecule (ICAM)-1 on leukocyte extravasation in the mouse cremaster muscle. We also provide evidence that reflected light oblique transillumination (RLOT) microscopy of translucent specimens lends itself to visualizing phenomena of cell motility such as cell polarization, lamellipodium formation, or uropod retraction, thus enabling the study of cell motility within living tissues.

\section{Methods}

\section{Animals}

Experiments were performed on male mice aged 2-3 months. Wild-type C57BL/6 mice were obtained from Charles River (Sulzfeld, Germany). P-selectin-deficient and ICAM-1-deficient mice were originally purchased from Jackson Laboratory (Bar Harbor, Me., USA) and are now held in our facility. Animals were kept under standard laboratory conditions and allowed free access to animal chow and tap water. All experiments were performed according to German legislation on the protection of animals.

\section{Surgical Preparation of Cremaster Muscles}

The surgical preparation was performed as originally described by Baez [12] with minor modifications. Mice were anesthetized using a ketamine/xylazine mixture $(100 \mathrm{mg} / \mathrm{kg}$ of ketamine and $10 \mathrm{mg} / \mathrm{kg}$ of xylazine) administered by intraperitoneal injection. The left femoral artery was cannulated in a retrograde manner for continuous blood pressure monitoring and the administration of substances to the cremaster vasculature. The right cremaster muscle was exposed through a ventral incision of the scrotum. The muscle was opened ventrally in a relatively avascular zone, using careful electrocautery to stop any bleeding, and spread over the transparent pedestal of a custom-made microscope stage. Epididymis and testicle were detached from the cremaster muscle and placed into the abdominal cavity. Throughout the procedure, the muscle was superfused with buffered saline. To minimize induction of inflammation by the surgical trauma, the muscle was handled as little as possible. After surgical preparation, which typically required $30 \mathrm{~min}$, the stage was transferred to the microscope and the temperature of the superfusion buffer was maintained at $37^{\circ} \mathrm{C}$ by an infrared heating lamp and a digital thermometer with a thermocouple small enough to allow for placement of the probe in close vicinity of the cremaster muscle.

\section{Intravital Microscopy}

The setup for intravital microscopy was centered around an Olympus BX50 upright microscope (Olympus Microscopy, Hamburg, Germany) equipped for stroboscopic fluorescence epi-illumination microscopy. Light from a 75 -watt xenon source was narrowed to a near monochromatic beam of a wavelength of $700 \mathrm{~nm}$ by a galvanometric scanner (Polychrome II; TILL Photonics, Gräfelfing, Germany) and directed onto the specimen via an FITC filter cube equipped with dichroic and emission filters (DCLP 500, LP515; Olympus). Microscopic images were obtained with Olympus water immersion lenses $(20 \times / \mathrm{NA} 0.5$ and $40 \times / \mathrm{NA} 0.8)(\mathrm{NA}=$ numerical aperture) and either recorded by a digital CCD camera (Imago; TILL Photonics) and stored on a personal computer using commercially available software (TILLvisION; TILL Photonics), or by an analog black and white CCD video camera (Cohu 4920; Cohu, San Diego, Calif., USA) and an analog video recorder (AG-7350-E; Panasonic, Hamburg, Germany). For real-time recordings (25 frames/s), the image acquisition cycle time was adjusted to $40 \mathrm{~ms}$. For time-lapse recordings, we chose cycle times between 1 and $5 \mathrm{~s}$, resulting in accelerations of 25-125 times over real time in the final movies, when displayed at 25 frames/s. Oblique illumination was obtained by positioning a mirroring surface (reflector) directly below the specimen and tilting its angle relative to the horizontal plane (fig. 1). The reflector consisted of a round cover glass (thickness 0.19-0.22 mm, diameter $11.8 \mathrm{~mm}$ ), which was coated with aluminum vapor (Freichel, Kaufbeuren, Germany) and brought into direct contact with the overlying specimen. By removing the reflector, the specimen could be transilluminated conventionally with a halogen light source via a 0.8-NA substage condenser (U-LWUCD; Olympus). To allow quantification of leukocytes rolling along and firmly adhering to the vessel wall, rhodamine $6 \mathrm{G}$ was injected intravenously as $20-\mu \mathrm{l}$ boluses of a $0.05 \mathrm{~g} / \mathrm{ml}$ solution shortly before every recording sequence. Intravitally stained blood cells were imaged at $525 \mathrm{~nm}$ excitation wavelength using a rhodamine filter set lacking an excitation filter. For the measurement of centerline blood flow velocity, green fluorescent microspheres $(6-\mu \mathrm{m}$ diameter; Molecular Probes, Leiden, The Netherlands) were injected via the arterial catheter, and their passage through the vessels of interest was recorded using the FITC filter cube under appropriate stroboscopic illumination (exposure $1 \mathrm{~ms}$, cycle time $10 \mathrm{~ms}, \lambda=488 \mathrm{~nm}$ ), integrating video images for sufficient time $(>80 \mathrm{~ms})$ to allow for the recording of several images of the same bead on one frame. Beads that were flowing freely along the vessels' centerline were used to determine blood flow velocity (see below).

\section{Quantification of Leukocyte Kinetics and Microhemodynamic} Parameters

For off-line analysis of parameters describing the sequential steps of leukocyte extravasation, we used either the CAMAS image analysis software (Dr. Zeintl, Heidelberg, Germany) for analog video recordings or the public domain software ImageJ (http://rsb.info. nih.gov/ij/) for digital image material. Rolling flux and firmly adherent cells were determined as described previously [13] and related to the vessel cross section and the luminal surface per $100-\mu \mathrm{m}$ vessel 
length, respectively. Emigrated cells were counted in an area covering $50 \mu \mathrm{m}$ on both sides of a vessel over $100-\mu \mathrm{m}$ vessel length. By measuring the distance between several images of one fluorescent bead under stroboscopic illumination, centerline blood flow velocity was determined. From measured vessel diameters and centerline blood flow velocity, apparent wall shear stress was calculated, assuming a parabolic flow velocity profile over the vessel cross section [14].

\section{Experimental Protocol}

Three postcapillary vessel segments were randomly chosen among those that were at least $150 \mu \mathrm{m}$ away from neighboring postcapillary venules and did not branch over a distance of at least $150 \mu \mathrm{m}$. After having obtained baseline recordings of leukocyte rolling, firm adhesion, and emigration as well as blood flow velocity in all three vessel segments, inflammation was induced by adding platelet-activating factor (PAF; Sigma-Aldrich, Deisenhofen, Germany) to the superfusion buffer at a final concentration of $100 \mathrm{n} M$. Measurements were repeated every $30 \mathrm{~min}$ for up to $120 \mathrm{~min}$, whereupon time-lapse recordings to visualize leukocyte motility during transendothelial and interstitial migration were performed. Finally, blood was drawn by cardiac puncture for the determination of systemic leukocyte counts and the animals were euthanized by an intra-arterial pentobarbital overdose.

\section{Image Processing}

Custom format digital images were converted to the TIFF format, contrast-enhanced with the Photoshop auto-contrast function and assembled to movies using Quicktime software (Apple Computer, USA).

\section{Statistical Analysis}

Groups were compared by one-way ANOVA followed by Student-Newman-Keuls test, using SigmaStat Software (Jandel Scientific, Erkrath, Germany). Mean values \pm SEM are given. Differences between groups reaching a $p$ value $<0.05$ were considered as significant.

\section{Results}

\section{Enhanced Image Contrast through RLOT}

To test the effect of RLOT on the contrast of microscopic images, we first visualized $15-\mu \mathrm{m}$ microspheres at defined angles (fig. 2a-d). The image obtained at a tilting angle of $0^{\circ}$ (fig. $2 \mathrm{~b}$ ) resembles the image of a microsphere transilluminated by the substage condenser (fig. 2a), while increasing the angle gradually enhanced a shadow cast, three-dimensional appearance of the microsphere (fig. 2c, d). To demonstrate the enhancement of image contrast of fine morphological details, a human buccal epithelial cell was imaged at $0^{\circ}$ (fig. 2e) and $15^{\circ}$ (fig. 2f) tilting angle. Subcellular structures that remain invisible in the first are clearly distinguishable in the latter. Comparison to conventional imaging methods utilizing interference phenomena such as phase contrast (fig. $2 \mathrm{~g}$ ) and differential interference contrast (fig. $2 \mathrm{~h}$ ) showed that

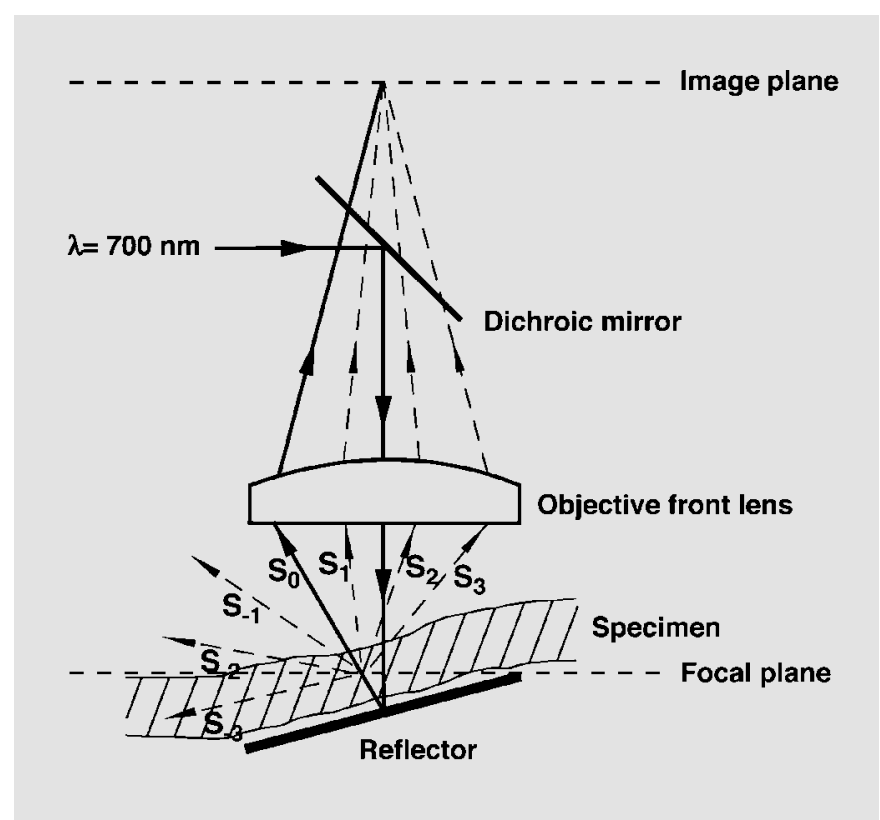

Fig. 1. Schematic, simplified representation of the light paths in RLOT microscopy: near-monochromatic light $(\lambda=700 \mathrm{~nm})$ is directed through the specimen onto the tilted reflector. The reflected light is diffracted by the specimen and several diffraction maxima emanate. While one sideband of diffracted light $\left(\mathrm{S}_{-1}-\mathrm{S}_{-3}\right)$ misses the objective front lens, several maxima from the other sideband $\left(\mathrm{S}_{1}-\mathrm{S}_{3}\right)$ as well as the undiffracted, direct light $\left(\mathrm{S}_{0}\right)$ enter the microscope light path and form an image at the image plane after interfering at the intermediate image plane (not shown).

RLOT yields images most similar to images obtained by the latter.

When imaging the mouse cremaster muscle with RLOT microscopy, the improvement with regard to image detail compared to the conventional brightfield image is immediately recognizable (fig. 3a, b). Even at low objective magnification $(20 \times)$, the morphology of endothelial cells or polarized extravasated leukocytes becomes discernible (fig. 3c). At higher objective magnification $(40 \times)$, even fine cellular protrusions such as filopodia of migrating leukocytes can be identified (fig. $3 \mathrm{~d}-\mathrm{g}$, see also online supplemental material). The optimum tilting angle of the reflector had to be adjusted for different objectives to obtain sufficient exclusion of one sideband of diffracted light. The angle depends on the front lens diameter, working distance and numerical aperture of the objective used and was empirically determined to be approximately $10^{\circ}$ and $15^{\circ}$ for the $20 \times$ and the $40 \times$ lens, respectively. 

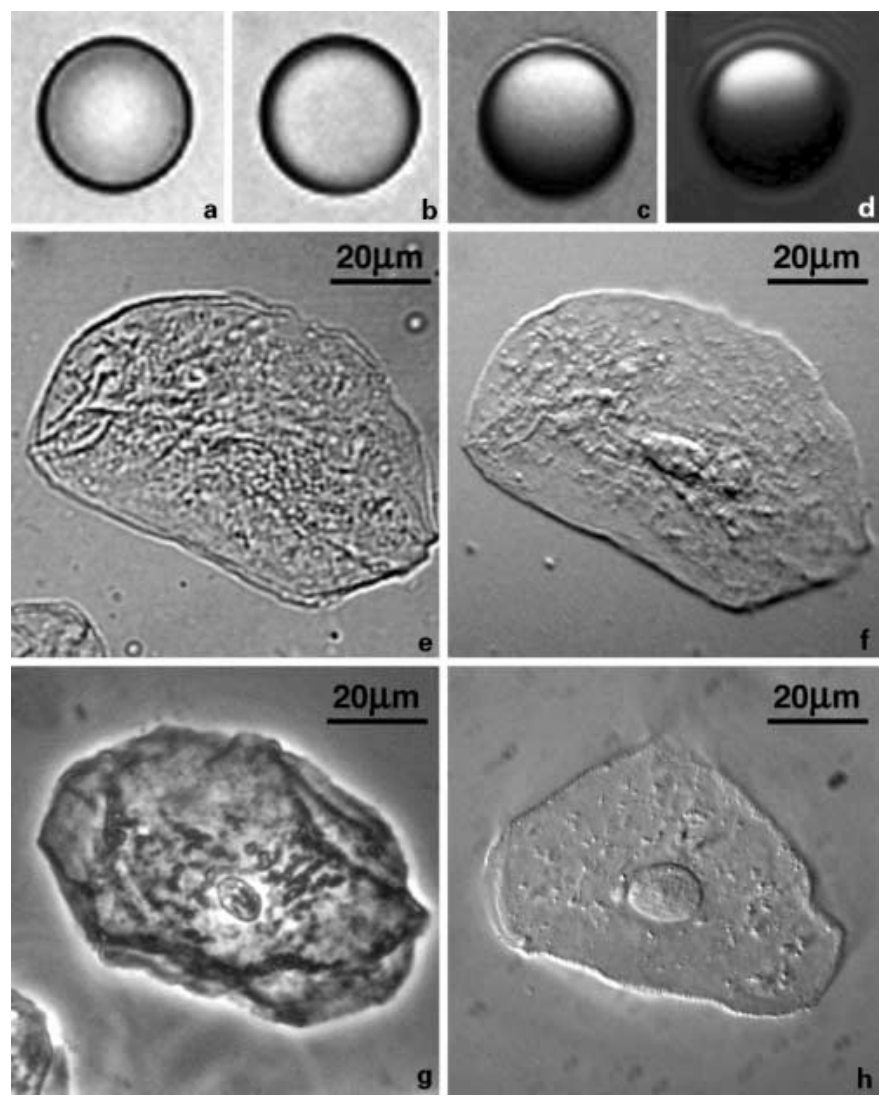

Fig. 2. Transmission (a) and RLOT micrographs (b-d) of $15-\mu \mathrm{m}$ diameter microspheres. The inclination of the reflector was set to $0^{\circ}$, $15^{\circ}$ and $30^{\circ}$ in $\mathbf{b}, \mathbf{c}$ and $\mathbf{d}$, respectively. The pseudo-three-dimensional appearance of objects when imaged with RLOT microscopy accounts for a clear depiction of subcellular structures as demonstrated for a human buccal epithelial cell imaged at a $15^{\circ}$ reflector inclination angle. The same cell in transmission mode (e) and as seen by RLOT microscopy (f) is shown. $\mathbf{g}$, $\mathbf{h}$ Images of buccal epithelial cells obtained with phase contrast and differential interference contrast, respectively, are shown.

\section{Quantification of Parameters of Leukocyte Extravasation}

Due to the mechanical trauma of the surgical preparation, a certain degree of inflammatory response of the cremaster muscle was present under baseline conditions. This probably accounts for at least some of the observed rolling and adhesive interactions of leukocytes with the endothelium of postcapillary cremasteric venules [15]. Under control conditions, the number of rolling interactions decreased and the number of adhesive interactions as well as of extravasated cells slightly increased over time (fig. $4 a-c)$. Application of an additional inflammatory
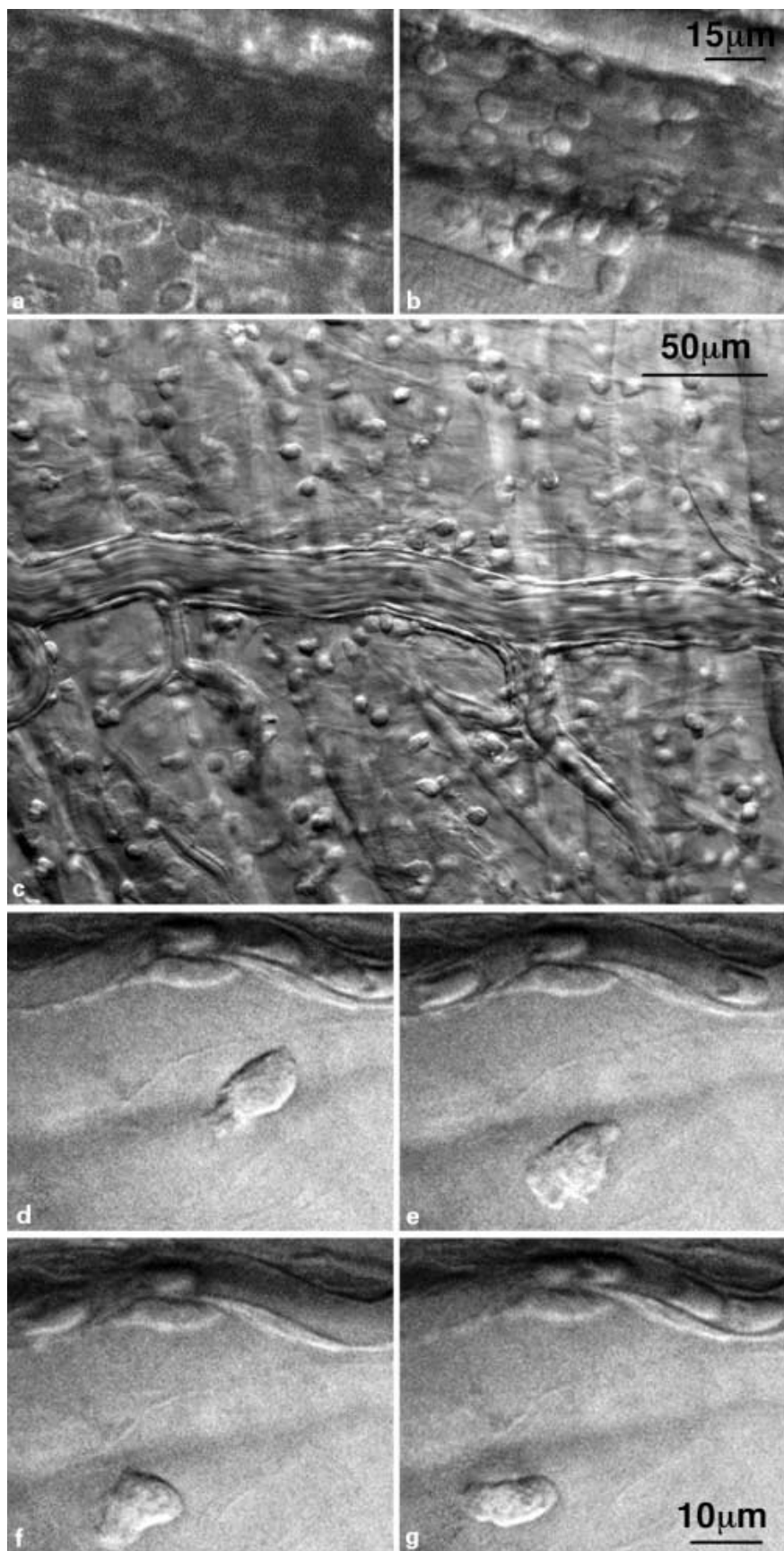

Fig. 3. Images from the mouse cremaster muscle after $>120$ min of continuous topical exposure to $10 \mathrm{n} M$ PAF. a, b Successive recordings of the same postcapillary vessel, using transmitted light (a) or ROLT (b) microscopy. Note the enhancement of image contrast obtained by RLOT. c A postcapillary venule surrounded by a dense inflammatory infiltrate is shown at low magnification. d, e Still images from a time-lapse recording of a leukocyte migrating in the vicinity of a capillary (also see on-line supplemental material for videos of time-lapse recordings). Because of the short exposure time of each frame $(1 \mathrm{~ms})$, the shape of fast-moving erythrocytes within the capillary lumen can be identified. 


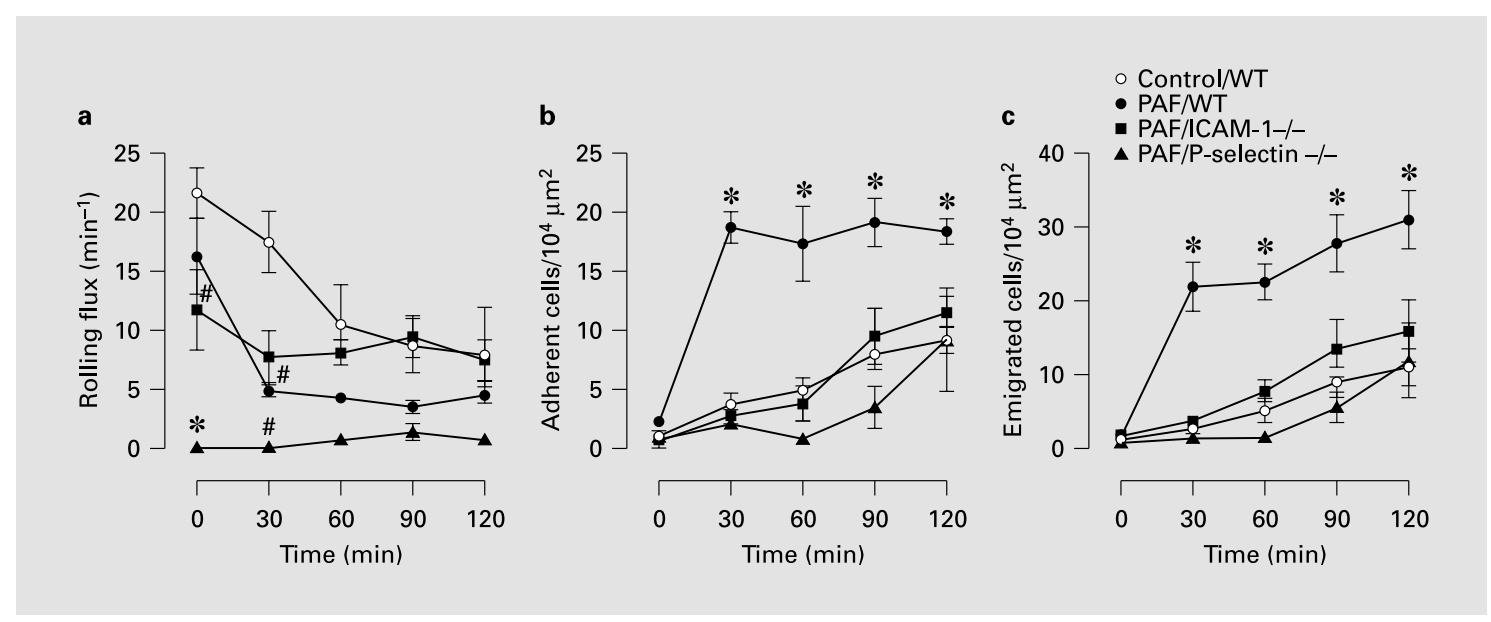

Fig. 4a-c. Quantitative analysis of parameters of leukocyte-endothelial cell interactions and leukocyte emigration. PAF was applied in the respective groups after baseline values had been obtained. ${ }^{*} \mathrm{p}<0.05 \mathrm{vs}$. control/WT; ${ }^{*} \mathrm{p}<$ 0.05 vs. all other groups. Values represent means \pm SEM. WT $=$ Wild-type.

stimulus by superfusion with PAF, however, induced a significant decrease in the number of rolling cells and an increase in the number of firmly adherent cells in wildtype animals within $30 \mathrm{~min}$. Moreover, the number of cells that had emigrated by this time had increased dramatically, indicating that firm adhesion was followed by emigration with little delay. After peaking at $30 \mathrm{~min}$, differences in all three parameters induced by PAF application in wild-type animals relative to untreated mice slowly diminished, possibly because the inflammatory response to the surgical trauma becomes dominant while PAFinduced effects may reach saturated levels. Of note, although application of PAF induced rapid and extensive edema formation in the tissue, which impaired visualization of rhodamine 6G-labeled leukocytes by fluorescence microscopy, image formation by RLOT microscopy was not noticeably impaired during the observation period of $2 \mathrm{~h}$. To demonstrate that intravital RLOT microscopy is able to detect defects in leukocyte migration to sites of inflammation when the initial steps in the transmigration cascade, rolling and adhesion, are blocked, additional experiments were performed in mice deficient in either P-selectin or ICAM-1. As expected, leukocyte rolling, firm adhesion, and emigration were virtually absent in Pselectin-deficient mice during the initial $60 \mathrm{~min}$ of PAF superfusion. In ICAM-1-deficient mice, however, only firm adhesion and emigration of leukocytes were impaired during the initial $60 \mathrm{~min}$ of PAF superfusion, while leukocyte rolling was rather unaffected.
Observation of leukocyte emigration by time-lapse video microscopy revealed that after arrest, leukocytes remained intravascularly for several minutes before emigrating. The time typically required for the subsequent penetration of the endothelium was in the range of only 1-3 min. Often, leukocytes would arrest abluminally after penetrating the endothelium, before vividly migrating in the extravascular space (see online supplemental material). Although we were not able to discern different leukocyte subpopulations in the interstitium by RLOT microscopy, extravasated leukocytes were $>90 \%$ polymorphonuclear leukocytes, as identified by immunohistochemical staining of fixed cremaster whole mounts (not shown). The time-lapse videos as well as the still images from such a recording shown in figure 3 demonstrate that RLOT microscopy, through significant enhancement of image contrast, facilitates the dynamic intravital visualization of leukocyte migration.

\section{Discussion}

Image formation of unstained specimens in optical microscopy occurs principally in two ways. Light-absorbing structures such as pigments yield contrast by reducing the wave amplitude of transmitted light. Since most biological specimens are devoid of significant amounts of pigment, contrast formation through absorption is poor. On the other hand, differences in refractive 
indices within tissues are ubiquitous and conversion of these refractive gradients into differences in image intensity, as has been realized by microscopic methods such as phase contrast [8], differential interference contrast [9], and Hoffmann modulation contrast [10], allows for the striking visualization of structural details in live, unstained specimens. However, despite their popularity in intravital imaging of Caenorhabditis elegans [16], these optical procedures have not been utilized in intravital imaging of mammals, most likely due to the requirement for polarizers and specialized filters and the need for their precise optical alignment, which is difficult in these cases due to space limitations. With intravital RLOT microscopy we present an optical technique that utilizes a relatively simple setup to generate the conditions necessary to convert optical gradients within a specimen into differences in intensity at the image plane, thereby enabling the visualization of morphological details in a thick turbid medium such as the interstitial tissue. The purpose of tilting the reflector underneath the specimen is to create a cone of reflected light that transmits the specimen in a direction oblique to the optical axis of the microscope. As illustrated in figure 1 , this preferentially excludes components of one sideband of diffracted light $\left(\mathrm{S}_{-1}-\mathrm{S}_{-3}\right)$ from the aperture of the objective lens. Thereby, the remaining sidebands $\left(\mathrm{S}_{1}-\mathrm{S}_{3}\right)$, which are phase-shifted against the undiffracted, direct light $\left(\mathrm{S}_{0}\right)$, can undergo constructive or destructive interference with the direct light at the intermediate image plane, creating an increase or decrease in light intensity at the image plane [17]. When light rays transmit a specimen in a direction parallel to the optical axis as in conventional brightfield transillumination, the sidebands of diffracted light are, in sum, $90^{\circ}$ out of phase with the direct light, which prevents the occurrence of constructive or destructive interference [8]. Variations in refractive index within tissue (such as cell membranes) create different degrees of phase modulation of diffracted light versus direct light, thereby determining light intensity at the image plane, where interference occurs. Since image formation by these means does not depend on light absorption, we used near-infrared light $(\lambda=700 \mathrm{~nm})$ to minimize absorption by intravascular hemoglobin or muscle myoglobin and to improve the penetration depth in the tissue [18].

In a previous study, MacDonald et al. [19] utilized a similar approach to generate high contrast images of the microcirculation in various solid organs such as spleen, liver, and pancreas. By resting their specimens on a transparent microscope stage of an inverted microscope and obliquely transilluminating it from above, using a cooled fiber-optic light source, they obtained images from organ areas near the surface facing the stage. This allows for transmitted light imaging of solid organs, because light from the focal plane does not need to pass through the whole thickness of the organ before entering the objective lens and therefore is scattered to a lesser extent. This method depends, however, on ample accessibility of the specimen from above and below, whereas in RLOT merely the reflector needs to be positioned underneath the specimen, extending the range of possible applications. Furthermore, usage of the objective lens for illumination and light collection provides the inherently optimized Köhler illumination, improving image quality.

Although the advent of multiphoton laser scanning microscopy [20] has provided a powerful tool for deep fluorescence tissue imaging, its logistic requirements and costs still prevent its widespread use as a routine microscopic technique. Also, multiphoton laser scanning microscopy depends on the presence of fluorescence markers to visualize tissue or cellular structures and is generally limited by its relatively slow scan rate. Intravital RLOT microscopy, on the other hand, as a form of widefield microscopy, can be performed at video rate and works on unstained samples. It can also easily be combined with widefield fluorescence microscopy, although the fluorescence of labeled cells in the interstitium does not provide much structural detail and merely allows for identification of labeled cells. This is presumably because out-offocus light is not excluded from image formation as is the case with the interference image, yielding blurry fluorescent images from objects within thick specimens. RLOT may therefore be useful for imaging fast dynamic processes or in instances where appropriate fluorescent markers are not available. Its main limitation is the requirement for a translucent specimen, under which a reflecting surface must be fitted. This condition is fulfilled for a number of living tissues such as rodent cremaster or tenuissimus muscles, the mesentery, or the hamster cheek pouch, making these tissues useful model tissues to study dynamic events within the extravascular compartment, as demonstrated for leukocyte migration in this case. Taken together, the enhancement of image contrast obtained by oblique transillumination with reflected light does not only enable the reliable quantitative assessment of leukocyte extravasation, but also the in vivo observation of cellular phenomena of cell migration, such as lamellipodium formation and uropod retraction with high morphological detail. 


\section{Acknowledgments}

We would like to thank Dr. Eckhart Hanelt for helpful discussions with regard to optophysical questions.

This work was supported by the European Commission grant QLG1-1999-01036 and DFG Graduate Program $438^{\circ}$ Vascular Biology in Medicine'. The data presented in this paper are part of the doctoral thesis of C.M.

\section{Supplemental Video Files}

Video 1. Overview of a postcapillary venular tree, taken at $20 \times$ objective magnification. In addition to several transendothelial migration events, numerous leukocytes can be observed during their migration within the cremasteric interstitium, displaying a characteristic probing behavior as well as polarization and depolarization during their migration. Events are accelerated $125 \times$ over real time and were recorded for $22 \mathrm{~min} 56 \mathrm{~s}$.
Video 2. Higher magnification $(40 \times$ objective lens) view of a single postcapillary venule, receiving a capillary. At this resolution, finer morphological details become discernible such as the segmentation and higher mobility of the lamellipodium. Events are accelerated $25 \times$ over real time and were recorded for $4 \min 10 \mathrm{~s}$.

Video 3 . A single leukocyte migrating in the vicinity of a capillary. For this recording, which lasted $2 \mathrm{~min}$ and $30 \mathrm{~s}$, the exposure time was deliberately set to $1 \mathrm{~ms}$. Under these conditions, the characteristic shape changes of red blood cells in the capillaries can also be observed. Acceleration is $25 \times$ over real time, objective magnification is $40 x$.

Video 4. In this video, initially an extravasating leukocyte comes into view, which has already largely penetrated but is still adhering to the endothelium with its uropod. Further downstream of the vessel, two different leukocytes can be observed during their diapedesis. While one of them leaves the vessel almost immediately, the other stays in the vicinity of the vessel for some time. The video is accelerated $125 \times$ over real time and was taken using a $40 \times$ objective.

For further information please refer to http://www.karger.com/doi/10.1159/000073902

\section{References}

1 Springer TA: Traffic signals for lymphocyte recirculation and leukocyte emigration: The multistep paradigm. Cell 1994;76:301-314.

2 Von Andrian UH, Mackay CR: T-cell function and migration. Two sides of the same coin. $\mathrm{N}$ Engl J Med 2000;343:1020-1034.

3 Cliff WJ: The acute inflammatory reaction in the rabbit ear chamber with particular reference to the phenomenon of leukocytic migration. J Exp Med 1966;124:543-556.

4 Raud J, Lindbom L, Dahlen SE, Hedqvist P. Periarteriolar localization of mast cells promotes oriented interstitial migration of leukocytes in the hamster cheek pouch. Am J Pathol 1989; 134:161-169.

5 Werr J, Xie X, Hedqvist P, Ruoslahti E, Lindbom L: Betal integrins are critically involved in neutrophil locomotion in extravascular tissue in vivo. J Exp Med 1998;187:2091-2096.

6 McLean PG, Ahluwalia A, Perretti M: Association between kinin $\mathrm{B}(1)$ receptor expression and leukocyte trafficking across mouse mesenteric postcapillary venules. J Exp Med 2000; 192:367-380
7 Hickey MJ, Forster M, Mitchell D, Kaur J, De Caigny C, Kubes P: L-selectin facilitates emigration and extravascular locomotion of leukocytes during acute inflammatory responses in vivo. J Immunol 2000;165:7164-7170.

8 Zernike F: Phase contrast: A new method for the observation of transparent objects. Physica 1942;9:686-693.

9 Nomarski G: Microinterféromètrie différentiel à ondes polarisées. J Phys Radium 1955;16: S9.

10 Hoffman R, Gross L: The modulation contrast microscope. Nature 1975;254:586-588.

11 Kachar B: Asymmetric illumination contrast: A method of image formation for video microscopy. Science 1985;227:766-768.

12 Baez S: An open cremaster muscle preparation for the study of blood vessels by in vivo microscopy. Microvasc Res 1973;5:384-394.

13 Von Andrian UH, M'Rini C: In situ analysis of lymphocyte migration to lymph nodes. Cell Adhes Commun 1998;6:85-96.

14 Tangelder GJ, Slaaf DW, Muijtjens AM, Arts T, oude Egbrink MG, Reneman RS: Velocity profiles of blood platelets and red blood cells flowing in arterioles of the rabbit mesentery. Circ Res 1986;59:505-514.
15 Kunkel EJ, Jung U, Bullard DC, Norman KE, Wolitzky BA, Vestweber D, Beaudet AL, Ley $\mathrm{K}$ : Absence of trauma-induced leukocyte rolling in mice deficient in both P-selectin and intercellular adhesion molecule 1. J Exp Med 1996;183:57-65.

16 Thomas C, DeVries P, Hardin J, White J: Four-dimensional imaging: Computer visualization of 3D movements in living specimens. Science 1996;273:603-607.

17 Ellis GW: Advances in visualization of mitosis in vivo; in Dirksen E, Prescott DM, Fox CE (eds): Cell Reproduction. London, Academic Press, 1978, pp 465-476.

18 MacVicar BA: Infrared video microscopy to visualize neurons in the in vitro brain slice preparation. J Neurosci Methods 1984;12: 133-139.

19 MacDonald IC, Ragan DM, Schmidt EE, Groom AC: Kinetics of red blood cell passage through interendothelial slits into venous sinuses in rat spleen, analyzed by in vivo microscopy. Microvasc Res 1987;33:118-134.

20 Denk W, Strickler JH, Webb WW: Two-photon laser scanning fluorescence microscopy. Science 1990;248:73-76. 